\title{
A Simulation Design Based on the Six Quadrants Laser Detection System
}

\author{
Yan-mei Zhang \\ School of Information and Electronics \\ Beijing Institute of Technology \\ Beijing,China \\ zhangym0726@163.com \\ Fei Xu \\ School of Information and Electronics \\ Beijing Institute of Technology \\ Beijing, China \\ promisefy@126.com
}

\author{
Xu Liu \\ School of Information and Electronics \\ Beijing Institute of Technology \\ Beijing, China \\ liuxuos@163.com \\ Zhi-yin Niu \\ School of Information and Electronics \\ Beijing Institute of Technology \\ Beijing, China \\ niuzhiyin@126.com
}

\begin{abstract}
In order to achieve the best destruction effect, the signal processing circuit of six quadrant laser detector will do the target identification and target discrimination according to the echo signal of each quadrant when the missile meets the target. In this way will make sure that the missile explodes in an appropriate position and an appropriate way. For detecting targets more precisely and sharpening the systematic antijamming ability, the way that lays aside two groups of detectors around the caliber is used. This article will prove the lowest velocity of whirl of shell body and design a simulation method to validate the feasibility of this way.
\end{abstract}

Keywords-the six quadrants laser detection system;antiinterference; the lowest velocity of whirl

\section{INTRODUCTION}

The laser fuze is a kind new fuze, used to detect or apperceive the target by a laser beam, and analyze the echo signal to determine the optimal burst time of missile[1].Six quadrants laser detection system includes two detectors, placed before and after the missile direction. Each of them has both transmitting and receiving subsystem over three quadrants, so the six signal detection subsystem consists of two branch, forward detection branch and backward detection branch. The forward one is the pre detonation branch, and the backward one is the detonate branch.

\section{The Working Principle Of Six Quadrant Laser DETECTION SYSTEM}

The system architecture is shown in Fig. 1.

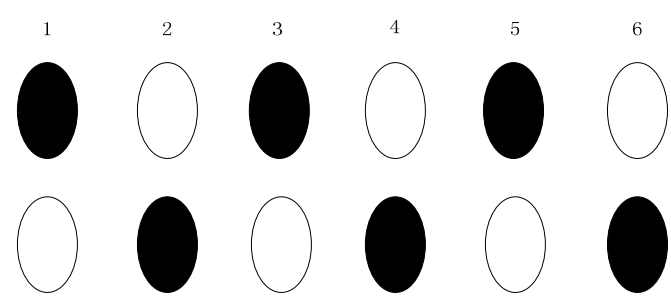

Figure 1. Circular Permutation of Detectors
In the figure, the black circles represent the transmitting terminals of the subsystem; the white circles represent the receiving terminals. The three sensors in 1, 3, 5 branch are used to transmit and receive the narrow pulse in forward branch. And sensors in 2,4,6 branch are for the backward branch. The laser fuze will actually work as the two branches receive the echo signals of the target, according to kinds of particular sequences and a certain time interval. When the two branches receive the signals almost at the same time, the target could be concluded as the interference such as clouds.

\section{The Design Of The Simulation}

\section{A. The Establishment of the Motion Model}

1) The Establishment of the Relative Motion Model Between Missile and Target. Fig.2 shows the relative horizontal movement model between missile and target. The $\mathrm{x}$-axis stands for relative horizontal distance between missile and target. The y-axis stands for relative vertical distance. The target's relative movement speed in vertical direction is zero, so we just take consideration of the movement in horizontal directions. In this direction, the target's relative speed is $v$, and the relative distance $d$ decreases gradually according to the time. 


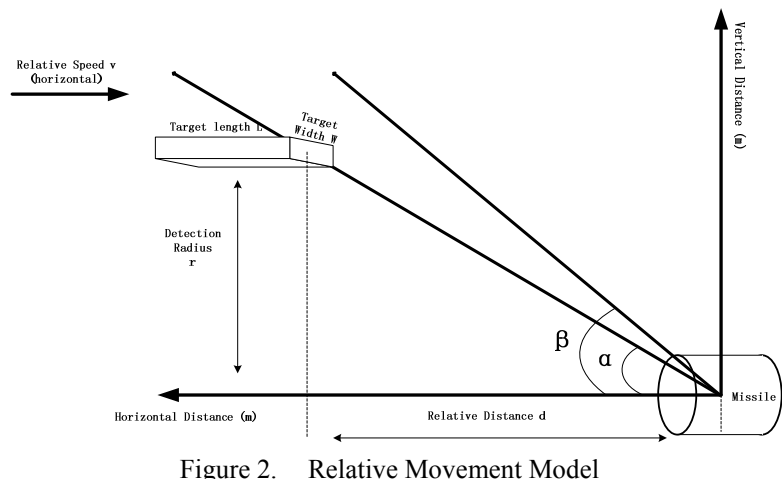

The formula of the distance $d$ between missile and target is as follows:

$$
d=d_{0}-v t
$$

$d_{0}$ stands for the initial distance;

$v$ stands for the relative horizontal speed;

$t$ stands for the passing time after the start of the simulation;

astands for the angle between the forward detector and the axle wire of the missile;

$\beta$ stands for the angle between the backward detector and the axle wire of the missile.

2) The Establishment of the Missile's Rotation Model. Fig. 3 shows the rotation model of the missile. The three circles represent the positions of forward detectors on the missile.

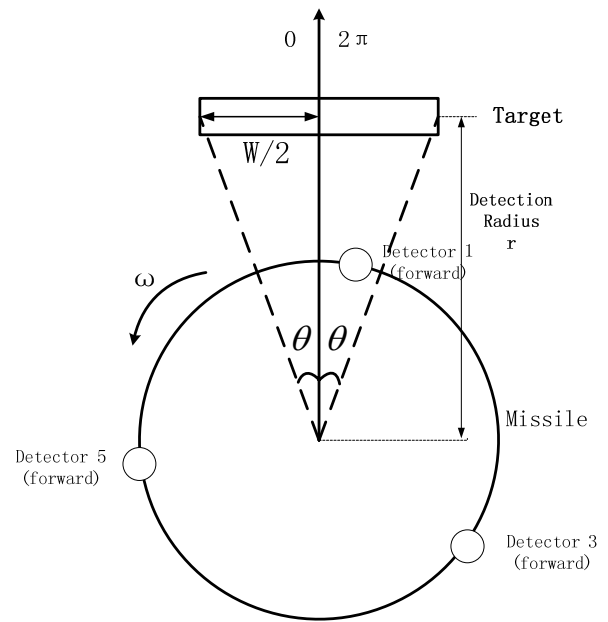

Figure 3. Missile's Rotation Model (back view)

We use the phase to express their positions,

$$
\sigma_{\text {phase }}=\sigma_{\text {phase } 0}+\omega t
$$

$\sigma_{\text {phase } 0}$ stands for the initial phase of the forward detector; $\omega$ stands for the angular speed of the missile; $t$ stands for the passing time after the simulation.

\section{B. Detection Strategies of Detectors}

When the detectors receive the echo signals from the target,they will determine the success of the detection, as is shown in the Fig. 4.

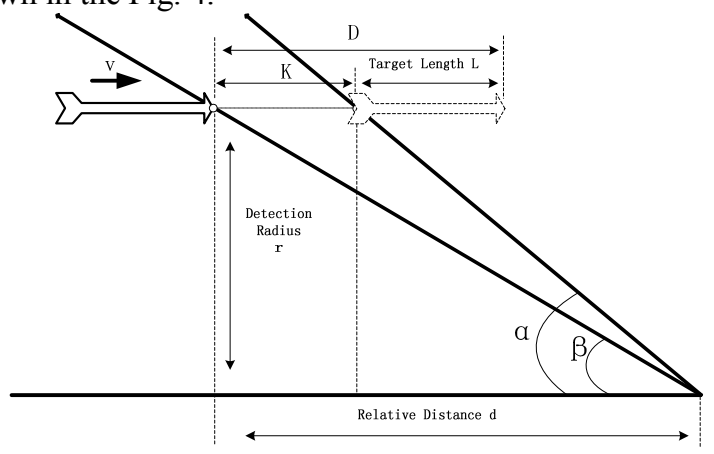

Figure 4. Judgment Strategy of Detection Zone

In the algorithm,there are two conditions which must be matched:

1) Judge Whether the Target in the Detection Zone. As is shown in Fig. 4, when the target and missile meet with each other in the horizontal direction, there are two conditions which must be matched, to determine the target is in the detection zone.

$$
\begin{gathered}
d \leq r \cdot \cot (\varphi) \\
d+L \geq r \cdot \cot (\varphi)
\end{gathered}
$$

In the formula,d stands for the length of the target;

$\varphi$ stands for the angle between detector and the axle wire of the missile.

When both (3) and (4) are right, the target is in the detection zone.

2) Judge WhetherTtarget is in the Phase of Detector. The missile is a cylinder, which has the symmetry in the phase of $0-2 \pi$. So no matter how the relative position between the target and missile are, we all judge the target is above the missile.

As is shown in Fig. 3,to judge the detector is in the direction of the target, the following conditions should be matched:

$$
0 \leq \sigma_{\text {phase }} \leq \theta
$$

or

$$
2 \pi-\theta \leq \sigma_{\text {phase }} \leq 2 \pi
$$

where

$$
\theta=\arctan \frac{W / 2}{r}
$$

When condition(5) or (6) is matched,it suggests the detector is in the direction of the target.

When the target is in the detection zone, and one of six detectors is in the direction of the target, we judge this detector detects the target successfully.

\section{The Minimum Rotation Speed of the Missile}

Fig. 5 shows how to judge the minimum rotation speed. 


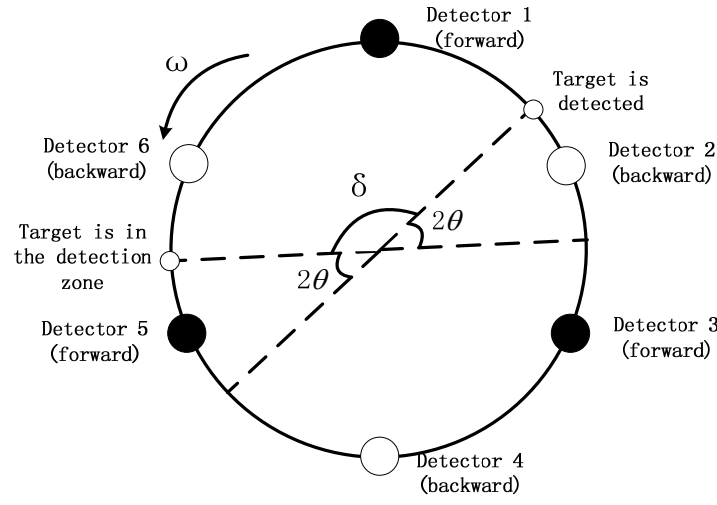

Figure 5. Judgment Strategy of Minimum Roation Speed

Suppose at the moment of the head of the target just in the detection zone, the detector 5(forward) doesn't detect it,in this case, in order to detect the target, there are two conditions must be matched:

First, detector 1 must catch the target successfully.

Second, at the moment of the target out of the detection zone, detector 2 just catches it.

In this case, we need the maximum time of target detection and the related rotation speed is the minimum one.

As shown in figure 4,the passing distance between the time intervals from the moment of head's stepping into the detection zone to the moment of tail's leaving the zone, is as follows:

where

$$
D=K+L
$$

$$
K=r \cdot \cot (\pi / 6)-r \cdot \cot (\pi / 4)
$$

and $L$ is the target's length.

So the passing time is,

$$
t=D / v
$$

Above all,as is shown in Fig. 5, the maximum angle in this time interval is,

$$
\delta=\pi-2 \theta
$$

so the minimum rotation speed is,

$$
\omega_{\min }=\delta / t
$$

And substitute (7),(8),(9),(10),(11) into (12),we can get

$$
\omega_{\min }=\frac{\pi-2 \arctan \frac{W}{2 r}}{r \cdot \cot (\pi / 6)-r \cdot \cot (\pi / 4)+L} \cdot \frac{v}{2 \pi}
$$

\section{Phase Judgment Strategy}

We use two branches to judge the receiving signals of six quadrants, one branch is the pre detonation branch, and the other is the detonate branch. When the target is detected, the echo signals are received by sensors according to some particular order in the time interval, and the fuze gives out the pre detonation signal. When the two branches receive the signals almost at the same time, the target could be concluded as the interference such as clouds.

In order to detect the target effectively, the information of target should meet the following criteria:
- The detection of forward detector should be earlier than the detection of backward detector;

- the forward and backward detectors which successfully detect the target should be next to each other;

- $\Delta T$ shouldn't be almost zero, otherwise it would be the interference such as clouds.

- $\Delta T$ should be smaller than the maximum time interval $\Delta T_{\max }$, and

$$
\Delta T_{\max }=\frac{\pi / 3}{\omega_{\min }}=\frac{\pi / 3}{70 \pi}
$$

- Forward $\Delta t_{\text {forth }}$ and backward $\Delta t_{\text {back }}$ should be in the domain from zero to maximum interval,

$$
\begin{aligned}
\Delta t_{\text {forth_max }} & =\frac{2 R_{\text {forth_max }}}{c} \\
\Delta t_{\text {back_max }} & =\frac{2 R_{\text {back_max }}}{c}
\end{aligned}
$$

- The absolute value of the difference of $\Delta t_{\text {forth }}$ and $\Delta t_{\text {back }}$ should be in the next domain

$\left|\Delta t_{\text {forth }}-\Delta t_{\text {back }}\right|_{\text {min }} \leq\left|\Delta t_{\text {forth }}-\Delta t_{\text {back }}\right| \leq\left|\Delta t_{\text {forth }}-\Delta t_{\text {back }}\right|_{\text {min }}$

where,

$$
\begin{aligned}
& \left|\Delta t_{\text {forth }}-\Delta t_{\text {back }}\right|_{\text {max }}=\left|\frac{2 R_{\text {forth_max }}-2 R_{\text {back_max }}}{c}\right| \\
& \left|\Delta t_{\text {forth }}-\Delta t_{\text {back }}\right|_{\text {min }}=\left|\frac{2 R_{\text {forth_min }}-2 R_{\text {back_min }}}{c}\right|
\end{aligned}
$$

where,

$\Delta t_{\text {forth }}$ is the time interval between transmitting and receiving laser pulse of forward detector; $\Delta t_{b a c k}$ is the time interval between transmitting and receiving laser pulse of backward detector; $\Delta T$ is the time interval between the receiving laser pulse of forward and backward detectors. Fig. 6.
The flow chart of phase judgment algorithm is shown in 


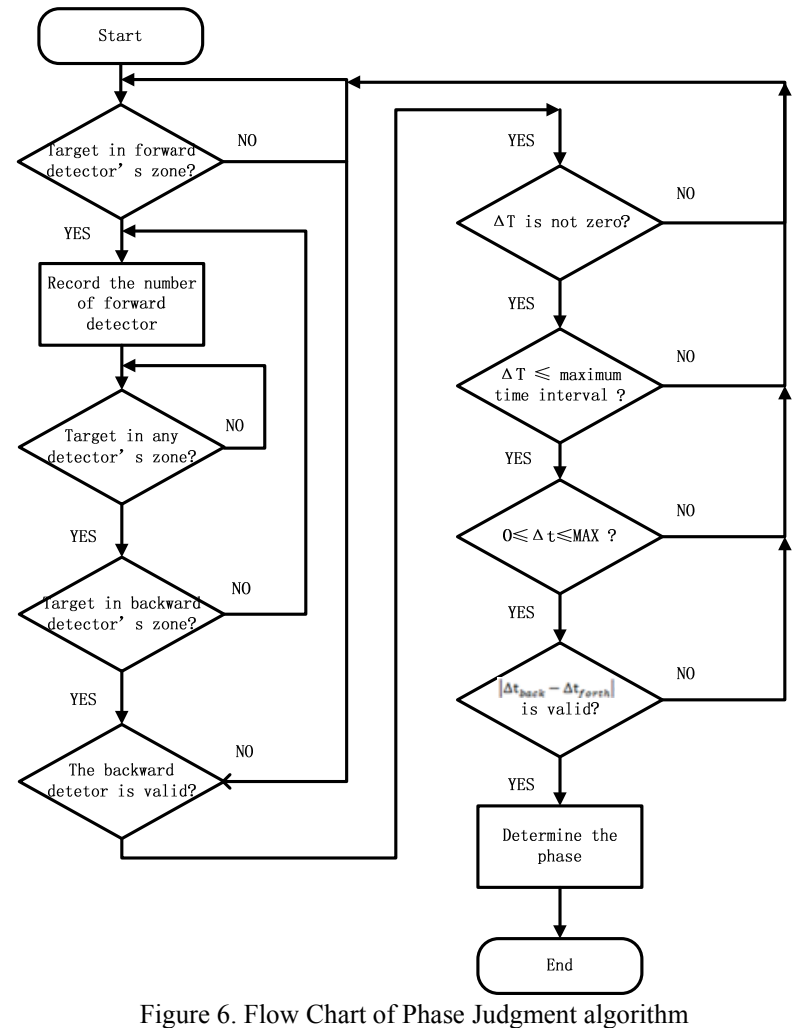

IV. The Analyse Of The Simulation's Result

A. The Analyse of Valid Result

When the target is in the phase 1,the result of six detectors' simulation is as follows:

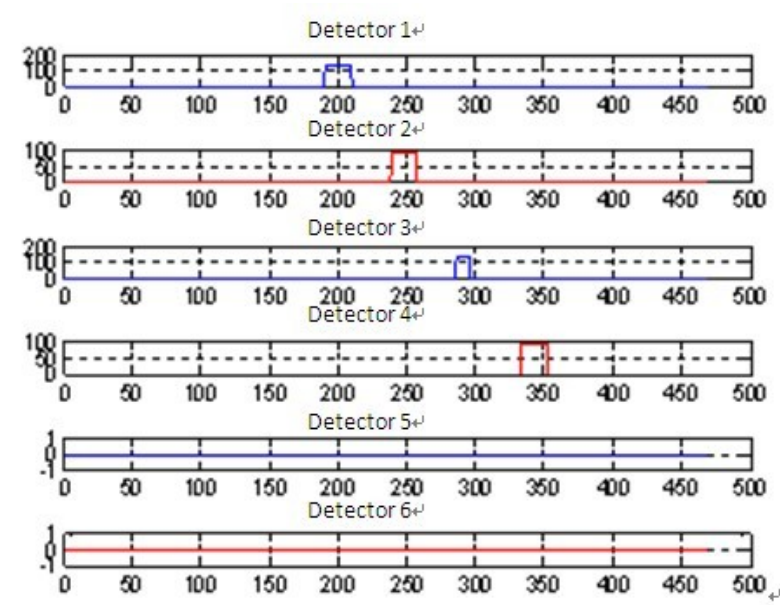

Figure 7. Six Detectors' Result when Target in Phase 1

Firstly, detector 1(forward) catches the target, then the detector 2 catches it, this case matches the condition of two detectors' adjoining. So we can get that the target is in phase 1.

\section{B. The Analyse of Invalid Result}

When the target is huge interference such as clouds, the simulation result of six detectors is as follows:

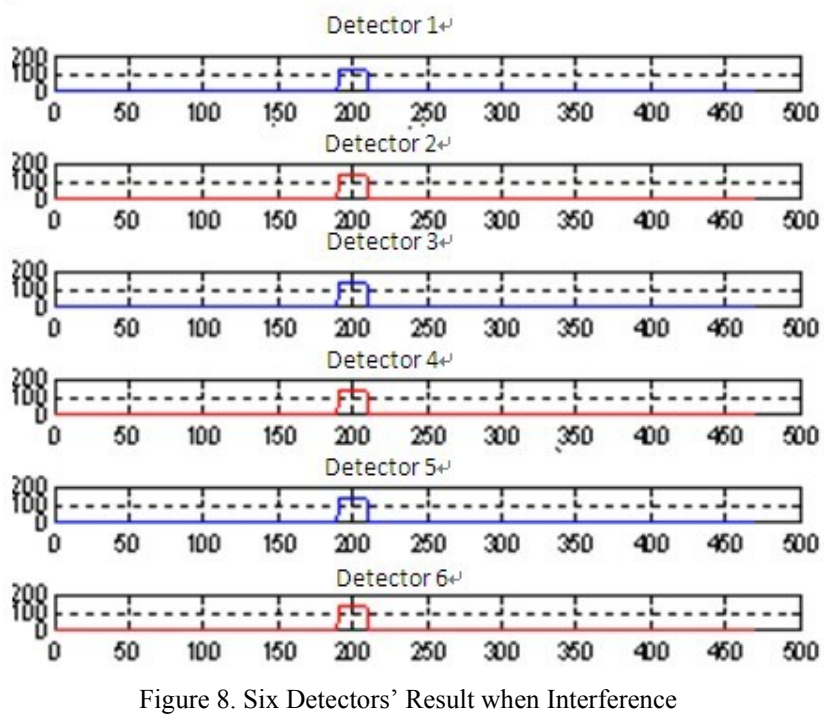

As is shown in Fig.8,six detectors find the target almost at the same time, so we regard it as the huge interference.

\section{CONCLUSION}

Above all the simulation and algorithm, in the case of missile and target's rendezvous in the horizontal direction, when the rotation speed of the missile is above $\omega_{\text {min }}$ and the receiving signals match the judgment strategies, the probability of forward and backward branches' detection can reach $100 \%$.

\section{REFERENCES}

[1] Chen Huimin,Li Ping,Li Kun.Scannerless imaging lidar detection techniques based on different systems[J]. Infrared and laser engineering, 2007,36(s1):219-222.

[2] Zhou Zhong-liang,Ying Jia-ju. Study on Image Processing Technology in Imaging Laser Detection System [J].Photonics and Optoelectronic (SOPO), 2010 Symposium on.

[3] YUAN Fei,MAO Zheng,LV Chun-hua,QIN Yue. Research on Simulation of Laser Detecting System[J].Computer Simulation,2012,29(2).

[4] CHEN Jin-ling, SHI Chua,XU Zheng-feng.Design of simulation software for lidar system, CHINA MEASUREMENT TECHNOLOGY, 2007, 33(5).

[5] WU Lin,ZHOU Bing,HE Yong-qiang,GENG Biao.Modeling and simulation of near-infrared omnidirectional laser imaging detection system,INFRARED AND LASER ENGINEERING,2009,38(2).. 